\title{
Comparison of tiotropium bromide and combined ipratropium/salbutamol for the treatment of COPD: a UK General Practice Research Database 12-month follow-up study
}

\author{
*Jane Griffina, Sally Lee ${ }^{a}$, Maria Caiadoa, Steven Kesten ${ }^{b}$, David Price \\ ${ }^{a}$ Health Economics \& Outcomes Research, Boehringer Ingelheim Limited, Bracknell, Berkshire, RG12 8YS, UK \\ ${ }^{b}$ Corporate Department of M edical Affairs, Boehringer Ingelheim Limited, Ingelheim, Germany \\ c Professor of Primary Care Respiratory M edicine, Department of General Practice and Primary Care, University of Aberdeen, Scotland, UK
}

\section{Received 23rd January 2008; revised version received 19th February 2008; accepted 11th March 2008}

\begin{abstract}
Aims: To compare the effectiveness of the long-acting anticholinergic, tiotropium with ipratropium/salbutamol in reducing the risk of exacerbations and COPD-related referrals in patients with COPD.

Methods: Data were obtained from the General Practice Research Database (GPRD). Propensity score matching was used to balance prognostic covariates between treatment groups. Incidence rate ratios and 95\% confidence intervals during a 12-month follow-up period were estimated.

Results: 4193 patients (3385, tiotropium; 808, ipratropium/salbutamol) in the GPRD met the inclusion/exclusion criteria. Patients treated with tiotropium had more severe COPD than patients treated with ipratropium/salbutamol. Following propensity score matching, 1222 tiotropium-treated patients and 633 ipratropium/salbutamol-treated patients were included in the final analysis. Incidence rate ratios (95\% confidence intervals) were $0.74(0.64-0.85 ; p=0.0086)$ for exacerbations and $0.57(0.46-0.70 ; p=0.004)$ for COPD-related referrals/hospitalisations.

Conclusions: Tiotropium is associated with a reduced risk of exacerbations and COPD-related referrals and hospitalisation compared to combined ipratropium/salbutamol in patients with COPD.

(c) 2008 General Practice Airways Group. All rights reserved.

J Griffin, et al. Prim Care Resp J 2008; 17(2): 104-110.

doi:10.3132/pcrj.2008.00024
\end{abstract}

Keyw ords COPD, general practice database, hospitalisation, ipratropium/salbutamol, tiotropium

\section{Introduction}

Chronic obstructive pulmonary disease (COPD) is characterised by chronic airflow limitation that is slowly progressive and not fully reversible. ${ }^{1}$ The disease is complicated by frequent and recurrent acute exacerbations that occur with increasing severity $^{2-4}$ and contribute to declining lung function. ${ }^{5}$ Exacerbations are associated with considerable morbidity and are a major factor in disease-related healthcare costs; it is estimated that exacerbations account for $35 \%$ to $45 \%$ of the total cost of COPD treatment. ${ }^{4,6,7}$ Bronchodilation and pharmacotherapy for exacerbations remain particularly important in the multidisciplinary approach to COPD treatment.

For second-line treatment in patients who are still symptomatic following initial treatment with a short-acting bronchodilator, COPD guidelines issued by the National Institute for Health and Clinical Excellence (NICE) ${ }^{8}$ recommend either combined therapy with a short-acting anticholinergic agent and a short-acting $\beta_{2}$-agonist (for example, ipratropium/salbutamol) or treatment with a long-acting bronchodilator - either a long-acting $\beta$-agonist (salmeterol or eformoterol) or a long-acting anticholinergic agent such as tiotropium. Available clinical trial data suggest that long-acting

\footnotetext{
* Corresponding author: M iss Jane Griffin, Health Economics \& Outcomes Research, Boehringer Ingelheim Limited, Bracknell, Berkshire, RG12 8YS, UK. Tel: +44 1344741494 Fax: +44 1344741298 Email: jane.griffin@boehringer-ingelheim.com
} 
anticholinergic therapy may provide better patient outcomes when compared with short-acting anticholinergics. In a series of international multicentre clinical trials of bronchodilators at recommended inhaled doses, tiotropium (once daily) was compared with the short-acting anticholinergic ipratropium (four times daily), the long-acting $\beta_{2}$-agonist salmeterol (twice daily), and placebo (patient's usual care, excluding anticholinergics and long-acting $\beta_{2}$-agonists) ${ }^{9-15}$ tiotropium provided sustained bronchodilation and improvements in dyspnoea and health-related quality of life and was also associated with fewer exacerbations than ipratropium and placebo. However, currently there are no direct comparisons of anticholinergic/ $\beta_{2}$-agonist combination therapy versus longacting anticholinergic agents in patients with COPD.

Therefore, the primary objective of this study was to compare the relative efficacy of the long-acting anticholinergic tiotropium bromide (Spiriva ${ }^{\oplus}$, Boehringer Ingelheim Ltd) with the short-acting combination preparation of ipratropium/ salbutamol (Combivent ${ }^{\oplus}$, Boehringer Ingelheim Ltd) in terms of the risk of COPD exacerbations and COPD-related hospital referrals in patients diagnosed with COPD over a 12-month period. Data for the study were obtained retrospectively from the UK General Practice Research Database (GPRD). Salmeterol was not included as a comparator in this study because data from a large-scale prospective head-to-head comparison of salmeterol and tiotropium have already been published. ${ }^{11}$

\section{Methods}

Study design

The study evaluated the difference between tiotropium and ipratropium/salbutamol in terms of COPD-related outcomes during a 12-month follow-up period. A 12-month period was thought to be necessary to detect any measurable change in outcomes and to allow for seasonal changes in COPD and its related conditions.

All data were extracted retrospectively from the GPRD database. The GPRD is the world's largest computerised database of anonymised longitudinal clinical records from general practice, comprising 46 million patient-years of data collected from approximately 13 million UK-based patients (3.4 million active patient records). ${ }^{16}$ The GPRD is widely known internationally as a resource for pharmacoepidemiology and a variety of other public health applications, including disease incidence and prevalence, drug utilisation, pharmacoeconomics, and health service resource use. The GPRD contains anonymised patient clinical records collected from a group of general practitioners (GPs) who use clinical system software in their practices. Patients are identified by encrypted and unique codes which are only decodeable by the practice; practices are also distinguished by encrypted codes. Neither patient nor practice identities are revealed to researchers.
The study period was between September 2002 and June 2006. The date of first recorded prescription for tiotropium or ipratropium/salbutamol was the patient's study entry date. Patients had to have received the first prescription of study treatment not earlier than September 2002 and had at least one year of follow-up on the study treatment before the end of June 2006. The study was approved by the Independent Scientific Advisory Committee (ISAC) for MHRA database research (protocol number: 07_044R).

Analysis of data from the GPRD relies upon delineating specific codes used in the database. In this study, a total of 90 medical codes of indications related to COPD were reviewed by a UK physician specialising in respiratory medicine, and all 320 product codes of medications were reviewed by either a UK physician or qualified UK pharmacist.

Patient inclusion criteria

The baseline population was all patients included in the GPRD with a first time prescription for tiotropium or ipratropium /salbutamol. Patients had to have a clinical record of a COPD diagnosis (i.e. before receiving the first prescription of study medication or during the 12-month follow-up). The cohort was further refined by an age of 35 years or over at first prescription of study medication. Patients were included in the cohort if they had at least 12 months of standard follow-up on study medication (i.e. sufficient prescriptions to cover at least 330 days of treatment to avoid bias towards therapy with greater compliance) and at least 12 months of medical history prior to study entry. To avoid confounding by partial compliance to study medication, estimated drug daily doses had to be within the licensed dose range (i.e. tiotropium $18 \mathrm{mcg}$ once daily; ipratropium/salbutamol $160 \mathrm{mcg}$, i.e. two puffs four times daily) and 'as required' ('prn') dosing instructions were excluded. Patients were also excluded if they had received concomitant anticholinergic medication (tiotropium, ipratropium or ipratropium/salbutamol) during the 12 months of follow-up. Outcome measurements

The following COPD-related outcomes were used to evaluate potential treatment differences between tiotropium and ipratropium/salbutamol:

- Rate of exacerbations over the 12-month follow-up period. Exacerbations were defined as the addition of oral steroids or short-term antibiotics (less than four weeks' duration) to the patient's study medication, with an inclusion code relating to a lower respiratory tract infection or COPD. Instead of using symptoms to define exacerbations, combinations of diagnosis and treatments were used. In GPRD, symptoms are only recorded if a suitable diagnosis is not available in a Read code. Since respiratory-related codes are well-developed in the Read system, and oral steroids/antibiotics are commonly used to treat COPD exacerbations, we believed that combinations of treatments and diagnosis would most closely match the 
actual exacerbation events in GPRD.

- Rate of COPD-related hospital referrals and hospitalisations over the 12-month follow-up period.

Hospital referrals and hospitalisations were defined as consultations to the appropriate clinical specialties (Respiratory M edicine, Geriatrics, General Medicine, Accident and Emergency (A\&E), Medical Assessment Unit) for all COPD-related reasons.

We were not able to include the forced expiratory volume in one second $\left(\mathrm{FEV}_{1}\right)$ as an outcome measurement since spirometry was scarcely recorded in the GPRD. It reflects the reality that GPs do not routinely measure spirometry in COPD patients and that the decline in $\mathrm{FEV}_{1}$ may not be the deciding factor in switching treatments.

Data analysis

On first assessment, it was found that there were significant differences in baseline characteristics between the two cohorts, in that tiotropium was prescribed to patients with more severe COPD (i.e., patients with more exacerbations and COPD-related referrals at baseline) as compared to those patients on ipratropium/salbutamol. This was not unexpected as tiotropium is a long-acting anticholinergic and the more recent of the two study drugs to be launched.

Therefore, to balance the prognostic covariates between the two cohorts, propensity score matching based on a logistic regression model was used to identify patients with similar baseline characteristics. The propensity score is a method which allows matched samples to be constructed which balance multiple covariates in the absence of randomisation. Introduced in 1983 by Rosenbaum and Rubin, ${ }^{17}$ propensity score matching provides better estimation of treatment effect in observational studies where the allocation of intervention is not randomised, and is thus more reliable than standard statistical methods in this situation. ${ }^{18-20}$ The propensity score aggregates a (significant) number of variables into one score using the logistic regression or the discriminant analysis. The score is then used for the matching or stratification of the subjects. The aim is to acquire two treatment groups of patients whose baseline characteristics are similar enough for outcome comparison.

Logistic regression models were used to create a propensity score for each patient who met the inclusion/ exclusion criteria. All covariates listed below were used to fit logistic regression models. Patients identified by propensity score matching were included in the final cohort for the outcome evaluations.

Incidence rate ratios and 95\% confidence intervals (CI) comparing the incidence rates of outcomes for each study cohort during the 12-month follow-up period were estimated. Covariates

All available covariates known to have an effect on COPDrelated outcomes were included in the logistic regression model to calculate the propensity score. Covariates included age, body mass index, smoking status, co-morbidities (asthma, cardiovascular disease, diabetes and osteoporosis), presence/absence of exacerbations and COPD-related referrals during the 12 months prior to the initiation of study treatment, and medication history (use of a long-acting bronchodilator, long-acting bronchodilator/inhaled steroid combination, inhaled steroid, salbutamol, oral steroids, supplemental oxygen, theophylline or aminophylline).

We also corrected for the presence and exposure of concomitant medications between the treatment cohorts, and included medications that would have an impact on outcomes (i.e. inhaled/oral steroids, salbutamol and supplemental treatments). The number and percentage of patients with a concomitant prescription of a COPD medication during the followup period was assessed by drug class. Concomitant prescribing was defined as a prescription of that medication between two and 11 months following initiation of the study drug.

\section{Power calculation}

We anticipated that the number of COPD exacerbations found in a database study like this would be lower than would be found in a randomised clinical trial. Therefore the lower value for COPD exacerbation rate found in those trials reviewed by the Cochrane collaboration was used. ${ }^{15}$ We estimated that $5 \%$ of tiotro pium patients and $10 \%$ of ipratropium/salbutamol patients would have a COPD exacerbation during the 12-month followup period. To detect a $5 \%$ difference between the proportion of patients who had any exacerbation during the 12-month followup, a sample size of 620 patients per treatment group would give $90 \%$ power, and 473 patients per treatment group would give $80 \%$ power at the $\alpha=0.05$ level of significance (two-sided). Data management and statistical analysis were performed using $\mathrm{SAS}^{\circledast}$ version 8.02.

\section{Results}

A total of 4193 patients in the GPRD met the inclusion/ exclusion criteria - 3385 in the tiotropium cohort and 808 in the ipratropium/salbutamol cohort. Patients treated with tiotropium had significantly more co-morbidities, exacerbations and COPD-related referrals, and higher usage of respiratory medications during the 12 months prior to entering the study, than patients treated with ipratropium/salbutamol.

\section{Patient population at baseline}

Propensity score matching successfully adjusted for the imbalances in the two treatment cohorts, with 1222 tiotropium-treated patients and 633 ipratropium/salbutamoltreated patients identified for inclusion in the final comparative analysis of treatment regimens. Table 1 shows that the baseline characteristics of the two treatment groups were well matched. The patient population comprised $57 \%$ men and 
Table 1. Baseline patient characteristics.

Tiotropium $(n=1222)$

Ipratropium/Salbutamol $(n=633)$

\begin{tabular}{|c|c|c|c|c|}
\hline \multicolumn{5}{|l|}{ Patient Characteristics } \\
\hline \multicolumn{5}{|l|}{ Sex, $n(\%)$} \\
\hline Female & 527 & $(43.1)$ & 270 & $(42.7)$ \\
\hline Male & 695 & $(56.9)$ & 363 & $(57.4)$ \\
\hline Age, mean $(S D)$, years & 69.2 & $(9.6)$ & 69.1 & $(10.5)$ \\
\hline \multicolumn{5}{|l|}{ Smoking, n (\%) } \\
\hline Non-Smoker & 186 & $(15.2)$ & 97 & (15.3) \\
\hline Ex-Smoker & 498 & $(40.8)$ & 256 & $(40.4)$ \\
\hline Passive-Smoker & 0 & $(0)$ & 0 & $(0)$ \\
\hline Smoker & 530 & $(43.4)$ & 278 & (43.9) \\
\hline Smoking Unknown & 8 & $(0.7)$ & 2 & $(0.3)$ \\
\hline Body Mass Index, mean (SD) & 25.41 & $(4.85)$ & 25.57 & $(4.92)$ \\
\hline \multicolumn{5}{|l|}{ Co-morbidity History, n (\%) } \\
\hline Diabetes & 190 & $(15.6)$ & 97 & (15.3) \\
\hline Cardiovascular disease & 266 & $(21.8)$ & 139 & $(22.0)$ \\
\hline Asthma & 1079 & $(88.3)$ & 547 & (86.4) \\
\hline Osteoporosis & 0 & (0) & 0 & (0) \\
\hline \multicolumn{5}{|l|}{ Medical History, n (\%) } \\
\hline Inhaled steroids & 750 & $(61.4)$ & 394 & $(62.2)$ \\
\hline Oral steroids & 535 & $(43.8)$ & 270 & $(42.7)$ \\
\hline Long-acting beta agonist & 300 & $(24.6)$ & 150 & (23.7) \\
\hline Theophylline & 144 & $(11.8)$ & 68 & (10.7) \\
\hline Oxygen supplement & 59 & $(4.8)$ & 25 & $(4.0)$ \\
\hline Salbutamol & 550 & $(45.0)$ & 282 & $(44.6)$ \\
\hline Exacerbation $^{b}$ & 213 & $(17.4)$ & 120 & $(19.0)$ \\
\hline COPD referrals/hospitalisation & 88 & $(7.2)$ & 46 & $(7.3)$ \\
\hline
\end{tabular}

Abbreviations: COPD, chronic pulmonary obstructive disease; SD, standard deviation.

${ }^{a}$ At least one prescription or event.

${ }^{\mathrm{b}}$ Exacerbations were defined as the addition of oral steroids or short-term antibiotics (less than 4 weeks duration) to the patient's study medication, with an inclusion code relating to a lower respiratory tract infection or COPD.

$43 \%$ women, with a mean age of 69 years. Approximately $43 \%$ were tobacco smokers, while $15 \%$ had never smoked. Twenty-two per cent of them had cardiovascular disease and about $15 \%$ had a history of diabetes; none had osteoporosis. Nearly two-thirds of patients had received at least one prescription for inhaled steroids over the previous 12 months, and over $40 \%$ had received a prescription for oral steroids or salbutamol. At baseline, approximately $18 \%$ of patients had suffered at least one exacerbation and $7 \%$ had at least one COPD-related referral/hospitalisation.

Outcome measurements

Exacerbations and COPD-related referrals/hospitalisations

At 12-month follow-up, the relative risk of an exacerbation was $26 \%$ lower for patients treated with tiotropium compared with ipratropium/salbutamol (incidence rate ratio $0.74 ; 95 \% \mathrm{Cl} 0.64-0.85 ; \mathrm{p}=0.0086$ ) (Table 2). The mean number of exacerbations was 0.18 per patient for the tiotropium group and 0.26 per patient for the ipratropium/ salbutamol group - a $31 \%$ difference. Amongst all patients who had any exacerbation during the follow-up period, more than $90 \%$ of them had one or two exacerbations, although the proportion of patients with more than one exacerbation was numerically higher in the ipratropium/salbutamol group.

The risk of COPD-related hospital referral or hospitalisation at 12-month follow-up was also reduced significantly for patients treated with tiotropium compared with ipratropium/ salbutamol (incidence rate ratio $0.57 ; 95 \% \mathrm{Cl}$ 0.46-0.70; $p=0.004$ ). The proportion of tiotropium-treated patients with 
Table 2. Outcome measurements during the 12-month study periods.

\begin{tabular}{|c|c|c|c|c|c|}
\hline & $\begin{array}{c}\text { Tiotropium } \\
(n=1222)\end{array}$ & $\begin{array}{l}\text { Ipratropium/ } \\
\text { Salbutamol } \\
(n=633)\end{array}$ & $\begin{array}{r}\mathrm{Re} \\
(95 \%\end{array}$ & $\begin{array}{l}\text { lative Risk } \\
\text { 6 Confidence } \\
\text { Interval) }\end{array}$ & $\mathrm{p}$ value \\
\hline \multicolumn{6}{|l|}{ Exacerbations ${ }^{a}$} \\
\hline Patients with at least 1 exacerbation, $n$ (\%) & $161(13.2)$ & $113(17.9)$ & 0.74 & $(0.64-0.85)$ & 0.0086 \\
\hline Total no. exacerbations & 215 & 162 & & & \\
\hline treated with antibiotics & 117 & 86 & & & \\
\hline treated with oral steroids & 98 & 76 & & & \\
\hline M ean no. exacerbations/patient & 0.18 & 0.26 & & & \\
\hline \multicolumn{6}{|l|}{ No. patients with } \\
\hline 1 exacerbation & 120 & 76 & & & \\
\hline 2 exacerbations & 32 & 28 & & & \\
\hline 3 exacerbations & 6 & 7 & & & \\
\hline 4 exacerbations & 2 & 1 & & & \\
\hline 5 exacerbations & 1 & 1 & & & \\
\hline \multicolumn{6}{|l|}{ COPD-related referrals/hospitalisation } \\
\hline Patients with at least 1 COPD-related referral, $n(\%)$ & $55(4.5)$ & $50(7.9)$ & 0.57 & $(0.46-0.70)$ & 0.004 \\
\hline Total no. COPD-related referrals & 86 & 70 & & & \\
\hline \multicolumn{6}{|l|}{ No. patients with } \\
\hline 1 COPD-related referral & 35 & 34 & & & \\
\hline 2 COPD-related referrals & 12 & 12 & & & \\
\hline 3 COPD-related referrals & 5 & 4 & & & \\
\hline 4 COPD-related referrals & 3 & 0 & & & \\
\hline \multicolumn{6}{|l|}{ Concomitant use of medications } \\
\hline \multicolumn{6}{|l|}{ Inhaled steroids } \\
\hline at least 1 prescription, $\mathrm{n}(\%)$ & $630(51.6)$ & $394(62.2)$ & 0.83 & $(0.76-0.91)$ & 0.0001 \\
\hline total no. prescriptions & 5006 & 3203 & & & \\
\hline mean prescriptions/person & 4.10 & 5.06 & & & \\
\hline \multicolumn{6}{|l|}{ Oral steroids } \\
\hline at least 1 prescription, $\mathrm{n}(\%)$ & $460(37.6)$ & $271(42.8)$ & 0.88 & $(0.79-0.97)$ & 0.0313 \\
\hline total no. prescriptions & 1635 & 990 & & & \\
\hline mean prescriptions/person & 1.34 & 1.56 & & & \\
\hline \multicolumn{6}{|l|}{ Oxygen supplement } \\
\hline at least 1 prescription, $\mathrm{n}(\%)$ & $73(6.0)$ & $36(5.7)$ & 1.05 & $(0.80-1.38)$ & 0.84 \\
\hline total no. prescriptions & 560 & 237 & & & \\
\hline mean prescriptions/person & 0.46 & 0.37 & & & \\
\hline \multicolumn{6}{|l|}{ Salbutamol } \\
\hline at least 1 prescription, $\mathrm{n}(\%)$ & $525(43.0)$ & $177(28.0)$ & 1.54 & $(1.34-1.76)$ & 0.0001 \\
\hline total no. prescriptions & 4824 & 1166 & & & \\
\hline mean prescriptions/person & 4.0 & 1.8 & & & \\
\hline
\end{tabular}

Abbreviations: COPD, chronic obstructive pulmonary disease; SD, standard deviation.

a Exacerbations were defined as the addition of oral steroids or short-term antibiotics (less than 4 weeks duration) to the patient's study medication, with an inclusion code relating to a lower respiratory tract infection or COPD.

at least one COPD-related hospital referral/hospitalisation was approximately half that of the ipratropium/salbutamol group. Most patients who required hospital referral/hospitalisation had one or two events over the 12-month follow-up period.
The proportion of patients with three or more COPD-related referrals/hospitalisations was low.

Concomitant use of respiratory medications

At 12-month follow-up, the risk of receiving at least one 
prescription for an inhaled or oral steroid was significantly greater for patients treated with ipratropium/salbutamol compared to tiotropium (Table 2).

Significantly more patients treated with tiotropium (43\%) received at least one prescription for salbutamol compared to ipratropium/salbutamol-treated patients $(28 \% ; p=0.0001$; Table 2). However, the difference in total salbutamol dose between the treatment groups was not significant. Salbutamol was part of the treatment regime for patients in the ipratropium/salbutamol group - patients treated with ipratropium/salbutamol were already receiving $800 \mathrm{mcg}$ salbutamol per day without separate prescriptions so that the total amount of $\beta_{2}$-agonist received was greater in the ipratropium/salbutamol group.

The risk of receiving supplementary oxygen therapy did not differ between the treatment groups (Table 2).

\section{Discussion}

In the absence of a randomised head-to-head comparison of tiotropium and ipratropium/salbutamol, this retrospective study used data in the GPRD on patients with COPD to conduct a matched cohort analysis. The data collected enable a comparison of the efficacy of these medications in terms of their ability to reduce exacerbations and COPD-related hospitalisation.

Prior to propensity score matching, tiotropium-treated patients had significantly more co-morbidities, exacerbations and COPD-related hospital referrals, and higher usage of respiratory medications in the 12 months prior to entering the study, than patients receiving ipratropium/salbutamol. This suggests that patients in the tiotropium group had more severe COPD than those treated with ipratropium/salbutamol - based on available information for severity assessment (i.e. without $\mathrm{FEV}_{1}$ ) - and that tiotropium, as a long-acting anticholinergic agent, may be preferentially selected for the more 'difficult-to-treat' patients.

We did not exclude patients with a diagnostic label of asthma, and as high as $85 \%$ of the patients meeting all inclusion criteria had records of asthma. How ever, we suspect that the majority of the asthma labels were a transitional diagnosis prior to the correct diagnosis of COPD. A study conducted within 1003 UK primary care practices highlighted the insecurity of the diagnostic process in primary care by showing that more than half of patients with a diagnostic label of asthma had a change in diagnosis, the change being mainly to a diagnosis of COPD. ${ }^{21}$ Similarly, it is unlikely that patients labelled as having COPD had a change in diagnosis to asthma. ${ }^{21}$ The diagnosis of COPD in our patient population can be further confirmed by their age and smoking status. The average age of the patients was over 69 and none of our patients was under the age of 38 . Smoking was also common amongst our patient population; over $84 \%$ of all patients had smoked or were still smoking. Given that a smoking history is often under-reported by patients to their GPs, we are certain that very few patients in our study had never smoked. If we had excluded patients with a label of asthma, the remaining $15 \%$ of patients would likely have been unrepresentative of COPD patients treated in primary care in the UK.

Based on the two groups of patients matched at baseline using propensity scores, the results of this study showed that tiotropium resulted in better disease-related outcomes; after 12 months, tiotropium was associated with a significantly reduced risk of exacerbation, COPD-related referral, and hospitalisation for COPD patients, as compared with ipratropium/salbutamol. The relative risk of COPD-related referral or hospitalisation was $43 \%$ lower for patients treated with tiotropium compared with ipratropium/salbutamol. The results are consistent with the only randomised controlled comparison of tiotropium versus usual care to be performed in a broad primary care population. ${ }^{22}$

In order to eliminate the possibility that the lower exacerbation rate and lower rate of COPD-related referrals found in patients treated with tioptropium was a result of the increasing use other respiratory medications, we assessed the concomitant use of respiratory medication during the 12month follow-up. The results indicated that the risk of receiving a prescription for inhaled or oral steroids during follow-up was significantly reduced for patients treated with tiotropium compared to ipratropium/salbutamol.

In addition to the clinical benefit of tiotropium as compared with ipratropium/salbutamol, tiotropium only requires one administration per day compared to four administrations per day for ipratropium/salbutamol. In addition, tiotropium has consistently demonstrated improvements in dyspnoea, health-related quality of life, exercise capacity, and exacerbation rates. ${ }^{9-15}$

In this retrospective study using "real-life" data, tiotropium use was associated with significantly better disease outcomes in all measures investigated when compared to ipratropium/ salbutamol. Tiotropium appears to be the better treatment choice when both tiotropium and ipratropium/salbutamol are considered to be appropriate treatment for the patient.

\section{Limitations}

One possible limitation of this study is the inclusion of patients who had been treated with tiotropium or ipratropium/salbutamol for at least a year; consequently one could argue that the study findings may not apply to patients who receive these medications for less than a year and that the data does not apply to all patients initiated on these therapies. Furthermore, as for any database analysis, data retrieval from the GPRD is limited to the variables contained on the database. Indicators such as $\mathrm{FEV}_{1}$ and MRC dyspnoea score, which have been traditionally used to assess COPD severity, are not available or not recorded at the right time- 
points to allow outcome comparison. Lack of spirometry measurements in COPD patients is, however, a common problem in UK general practice. Based on the same GPRD data, it has been reported that only $25.2 \%$ of COPD patients had an available current pulmonary function test. ${ }^{23}$

It should also be noted that the GPRD includes information on drug prescriptions which was used as a proxy measure for drug use in the present study. It cannot be assumed from this information that prescriptions were filled or that patients were compliant with their medication.

In contrast to randomised controlled trials (RCTs), GPRD studies provide real life data and highlight issues which may not be discovered in RCTs. Examples included the diagnostic confusion between COPD and asthma, the fact that $\mathrm{FEV}_{1}$ is not routinely measured or recorded, and the self-reporting of smoking status. Observational studies such as this cannot replace RCTs, but they provide complementary information which can provide a different understanding of the disease and clinical practice.

Not having sufficient information regarding the management of patients in secondary (hospital) care is another limitation of the GPRD; however, we believe this will be improved in the future as GPRD is introducing the new feature of linking hospital data to their current system.

\section{Conclusions}

Our findings indicate that 12 months of tiotropium use in patients with COPD is associated with a reduced risk of exacerbations and COPD-related hospital referrals compared to using combined ipratropium and salbutamol.

\section{Conflict of interest declaration}

JG, SL and MC are employees of Boehringer Ingelheim Ltd UK. SK is an employee of Boehringer Ingelheim Corporate. DP has no shares in pharmaceutical companies. He has received speaker's honoraria for speaking at sponsored meetings from the following companies marketing respiratory products: 3M, Altana, Astra Zeneca, BI, GSK, IVAX, MSD, Novartis, Pfizer, Schering-Plough. He has received honoraria for advisory panels with; 3M, Altana, Astra Zeneca, Bl, GSK, IVAX, M SD, Novartis, Pfizer, Schering-Plough. He or his research team have received funding for research projects from: 3M, Altana, Astra Zeneca, Bl, GSK, IVAX, MSD, Novartis, Pfizer, Schering-Plough, Viatris.

\section{Acknow ledgements}

This study was funded by Boehringer Ingelheim, Bracknell, UK. We would also like to thank Dr Sue Libretto for editing and preparing the manuscript, and Margaret Ross for assistance with submission.

\section{Contributions}

All authors inputted into the study design, analysis plan, study report and final manuscript. In addition Sally Lee undertook the data analysis and David Price reviewed the coding and drug lists. Presented as a poster and oral presentation at the British Thoracic Society Winter M eeting, London, 5-7 December 2007. Publication: Griffin J, Lee S, Caiado M, Williams T, Kesten S, Price D. Comparative assessment of continuous treatment for diagnosed COPD with tiotropium bromide (Spiriva), combined ipratropium and salbutamol (Combivent ${ }^{\circledR}$ ). A 12-month follow-up study in the General Practice Research Database.

\section{References}

1. Pauwels RA, Buist S, Calverley PMA, et al. Global initiative for chronic obstructive Lung Disease. Bethesda: National Heart, Lung \& Blood
Institute/World Health Organisation, 2003.

2. Dewan NA, Fafique $S$, Kanwar B, et al. Acute exacerbation of COPD, factors associated with poor treatment outcome. Chest 2000;117:662-71.

3. Rodriguez-Roisin R. Toward a consensus definition for COPD exacerbations. Chest 2000;117:398S-401S.

4. Andersson F, Borg S, Jansson SA, et al. The costs of exacerbations in chronic obstructive pulmonary disease (COPD). Respir Med 2002;96:7000-08.

5. Donaldson GC, Seemungal TA, Bhowmik A, et al. Relationship between exacerbation frequency and lung function decline in chronic obstructive pulmonary disease. Thorax 2002;57:847-52.

6. M iravitlles M, M urio C, Guerrero R, et al. Costs of chronic bronchitis and COPD: a 1-year follow-up study. Chest 2003;123:784-91.

7. Oostenbrink JB, Rutten-van Mölken MPMH. Resource use and risk factors in high cost exacerbations of COPD. Respir Med 2004;98:883-91.

8. Pearson M. Chronic obstructive pulmonary disease: Nation clinical guideline on management of Chronic obstructive pulmonary disease in adults in primary and secondary care. Thorax 2004;59 (Suppl 1):1-232.

9. Vincken W, van Noord JA, Greefhorst AP, et al. Improved health outcomes in patients with COPD during 1 year's treatment with tiotropium. Eur Respir J 2002;19:209-16.

10. Casaburi R, Mahler DA, Jones PW, et al. A long-term evaluation of once-daily inhaled tiotropium in chronic obstructive pulmonary disease. Eur Respir ] 2002; 19:217-24.

11. Brusasco V, Hodder R, Miravitlles $M$, et al. Health outcomes following treatment for six months with once daily tiotropium compared with twice daily salmeterol in patients with COPD. Thorax 2003;58:399-404.

12. O'Donnell DE Flüge $T$, Gerken F, et al. Effect of tiotropium on lung hyperinflation, dyspnoea and exercise tolerance in COPD. Eur Respir J 2004;23:832-40.

13. Niewoehner DE, Rice K, Cote $C$, et al. Prevention of exacerbations of chronic obstructive pulmonary disease with tiotropium, a once-daily inhaled anticholinergic bronchodilator. Ann Intern Med 2005;143:317-26.

14. Maltais F, Hamilton A, Marciniuk D, et al. Improvements in symptom-limited exercise performance over $8 \mathrm{~h}$ with once-daily tiotropium in patients with COPD. Chest 2005;128:1168-78.

15. Barr RG, Bourbeau J, Camargo CA, Ram RFS. Tiotropium for stable chronic obstructive pulmonary disease. Cochrane Database of Systematic Review 2005 Issue 2. Art. No.: CD002876.

16. GPRD. Available at: www.gprd.com/home/ [Accessed: 26 October 2007].

17. Rosenbaum PR, Rubin DB. The central role of the propensity score in observational studies for causal effects. Biometrika 1983;70:41-55.

18. Rubin DB. Estimating causal effects from large data sets using propensity scores. Ann Intern Med 1997;127:757-63.

19. Gu XS, Rosebaum PR. Comparison of multivariate matching methods: structures, distances, and algorithms. J Comput Graph Stat 1993;2:405-20.

20. Drake C. Effect of misspecification of the propensity score on estimators of treatment effect. Biometrics 1993;49:1231-6.

21. Pearson M, Ayres JG, Sarno M, Massey D, Price D. Diagnosis of airway obstruction in primary care in the UK: the CADRE (COPD and Asthma Diagnostic/management Reassessment) programme 1997-2001. Int J Chron Obstruct Pulmon Dis 2006;1(4):435-43.

22. Freeman $D$, Lee $A$, Price $D$, et al. Efficacy and safety of tiotropium in COPD patients in primary care - the SPiRiva Usual CareE (SPRUCE) study. Respir Res 2007;8:45.

23. Soriano JB, Maier WC, Visick G, Pride NB. Validation of general practictionerdiagnosed COPD in the UK General Practice Research Database. Eur J Epidemiol 2001;17(12):1075-80.

24. Jara M, Lanes SF, Wentworth C 3rd, May C, Kesten S. Comparative safety of long-acting inhaled bronchodilators: a cohort study using the UK THIN primary care database. Drug Saf 2007;30(12):1151-60.

Available online at http://w w w.thepcrj.org 